United States of America; ${ }^{2}$ Mayo Clinic, Division of Rheumatology, Rochester, United States of America; ${ }^{3}$ Mayo Clinic, Department of Quantitative Health Sciences, Rochester, United States of America

Background: Patients with rheumatoid arthritis (RA) have an increased burden of multimorbidity. Racial/ethnic disparities have also been associated with an increased burden of multimorbidity.

Objectives: We aimed to compare multimorbidity among different racial/ethnic groups and geographic regions of the US in patients with RA and comparators without RA.

Methods: We used a large longitudinal, real-world data warehouse with de-identified administrative claims for commercial and Medicare Advantage enrollees, to identify cases of RA and matched controls. Cases were defined as patients aged $\geq 18$ years with $\geq 2$ diagnoses of RA in January 1, 2010 - June 30,2019 and $\geq 1$ prescription fill for methotrexate in the year after the first RA diagnosis. Controls were persons without RA matched 1:1 to RA cases on age, sex, census region, calendar year of index date (corresponding to the date of second diagnosis code for RA), and length of prior medical/pharmacy coverage. Race was classified as non-Hispanic White (White), non-Hispanic Black (Black), Asian, Hispanic, or other/unknown, based on self-report or derived rule sets. Multimorbidity (2 or more comorbidities) was defined using 25 chronic comorbidities from a combination of the Charlson and Elixhauser Comorbidity Indices assessed during the year prior to index date. Rheumatic comorbidities were not included. Logistic regression models were used to estimate odds ratios (OR) with $95 \%$ confidence intervals $(\mathrm{Cl})$.

Results: The study included 16,363 cases with RA and 16,363 matched non-RA comparators (mean age 58.2 years, $70.7 \%$ female for both cohorts). Geographic regions were the same in both cohorts: $50 \%$ South, $26 \%$ Midwest, $13 \%$ West, and $11 \%$ Northeast. Race/ethnicity was not part of the matching criteria and varied slightly between the cohorts: among RA (non-RA) patients, $74 \%$ (74\%) were White, $11 \%$ (9\%) Hispanic, 10\% (9\%) Black, 3\% (4\%) Asian, and 3\% (4\%) other/unknown. Patients with RA had more multimorbidity than non-RA subjects $(51.3 \%$ vs $44.8 \%)$. Multimorbidity comparisons across US geographic regions were similar in both cohorts, with comparable multimorbidity levels for patients in the West and Midwest and higher levels for those in the Northeast and South (Figure 1). Among the non-RA patients, $43.5 \%$ of Whites experienced multimorbidity, compared to $33.9 \%$ of Asians, $46.1 \%$ of Hispanics, and $58.4 \%$ of Blacks. These associations remained after adjustment for age, sex, and geographic region, with significantly lower multimorbidity among Asians (OR: 0.81; 95\% Cl: 0.67-0.99) and significantly higher multimorbidity among Hispanics (OR: $1.21 ; 95 \% \mathrm{Cl}: 1.07-1.37$ ) and Blacks (OR: $1.74 ; 95 \% \mathrm{Cl}$ : $1.54-1.97$ ), compared to Whites in the non-RA cohort. Among the RA patients, racial/ethnic differences were less pronounced; $50.6 \%$ of Whites, $42.8 \%$ of Asians, $48.8 \%$ of Hispanics, and $58.4 \%$ of Blacks experienced multimorbidity. Adjusted analyses revealed no significant differences in multimorbidity for Asians (OR: $0.88 ; 95 \% \mathrm{Cl}: 0.70-1.08$ ) and Hispanics (OR: $1.06 ; 95 \% \mathrm{Cl}: 0.95-1.19)$ and a less pronounced increase in multimorbidity among Blacks (OR: 1.32; $95 \% \mathrm{Cl}$ : 1.17-1.49) compared to Whites in the RA cohort.

Conclusion: This large nationwide study showed increased occurrence of multimorbidity in RA versus non-RA patients and in both cohorts for residents of the Northeast and South regions of the US. Racial/ethnic disparities in multimorbidity were more pronounced among patients without RA compared to RA patients. This indicates the effects of RA and race/ethnicity on multimorbidity do not aggregate. The underlying mechanisms for these associations require further investigation.

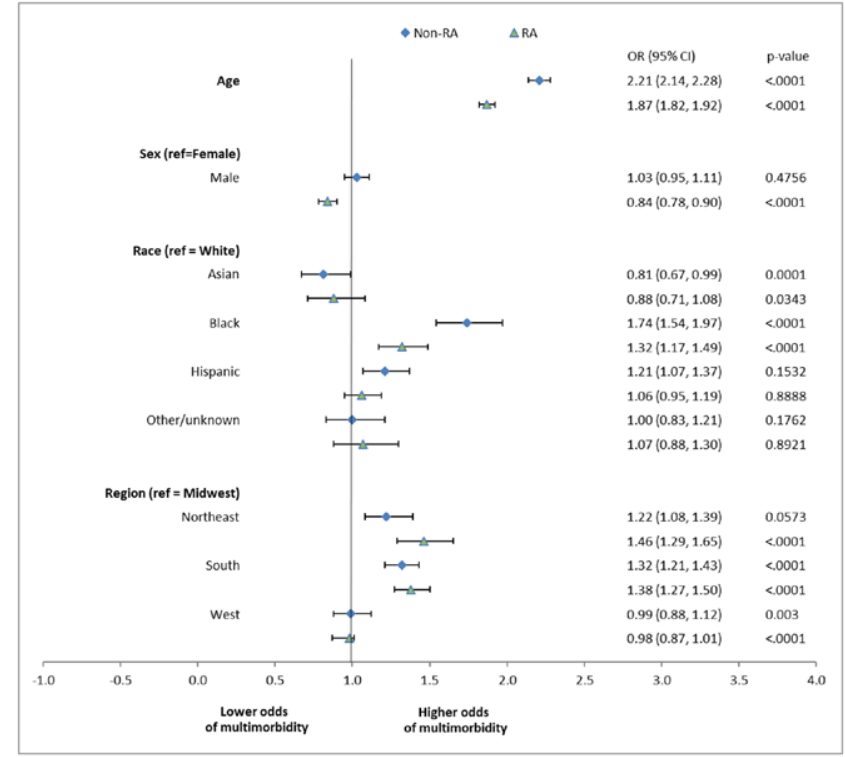

Figure 1. Logistic regression models comparing multimorbidity levels in RA and non-RA cohorts.

Disclosure of Interests: Hayley J. Dykhoff: None declared, Elena Myasoedova: None declared, Madeline Peterson: None declared, John M Davis III Grant/ research support from: Research grant from Pfizer, Vanessa Kronzer: None declared, Caitrin Coffey: None declared, Tina Gunderson: None declared, Cynthia S. Crowson: None declared.

DOI: 10.1136/annrheumdis-2021-eular.1610

\section{POS0024 \\ ESTIMATED PREVALENCE, INCIDENCE AND HEALTHCARE COSTS OF SJÖGREN'S SYNDROME IN FRANCE: A NATIONAL CLAIMS-BASED STUDY}

R. Seror ${ }^{1}$, L. Chiche ${ }^{2}$, G. Desjeux ${ }^{3}$, J. Zhuo ${ }^{4}$, B. Bregman ${ }^{5}$, V. VannierMoreau ${ }^{6}$, V. Devauchelle-Pensec ${ }^{7} .{ }^{1}$ Université Paris-Sud, AP-HP, Hôpitaux Universitaires Paris-Sud, N/A, Paris, France; ${ }^{2}$ Hôpital Européen Marseille, Unité de medicine interne et recherche clinique, Marseille, France; ${ }^{3}$ Sanoia, eHealth services, Aubagne, France; ${ }^{4}$ Bristol Myers Squibb, Worldwide Health Economics and Outcomes Research, Princeton, United States of America; ${ }^{5}$ Bristol Myers Squibb, Health Economics and Public Health, RueilMalmaison, France; ${ }^{6}$ Bristol Myers Squibb, Medical Affairs, France, RueilMalmaison, France; ${ }^{7} \mathrm{CHU}$ de Cavale Blanche, Rheumatology Department, Brest, France

Background: Sjögren's syndrome (SS) is a chronic, systemic autoimmune disorder characterised by oral and ocular dryness related to lymphocytic infiltration of exocrine glands. ${ }^{1-3}$ Extra-glandular manifestations may include fatigue, musculoskeletal pain and glomerulonephritis. ${ }^{1,3}$ SS can present as primary SS (pSS) 
or as SS associated with another autoimmune disorder (SS+AID). ${ }^{1}$ Reported prevalence of pSS in the French general population is $1.02-1.52$ cases/10,000 people. ${ }^{3}$

Objectives: To estimate prevalence, incidence and healthcare costs of SS in France using national health insurance claims data.

Methods: Claims from 2011-2018 from the Système National des Données de Santé (SNDS), comprised of data from $99 \%$ of the French population, were analysed. Patients (pts) with SS were identified using an algorithm. ${ }^{4}$ Inclusion criteria: International Classification of Diseases, Tenth Revision code M35.0 (Sicca syndrome [Sjögren]) or M35 (other systemic involvement of connective tissue), and $\geq 2$ drug reimbursements for SS-related treatments. Key exclusion criteria: amyloidosis, sarcoidosis, HIV, and head and neck radiotherapy. Denominators for estimated prevalence rates were derived from French population census data. Healthcare costs were based on Assurance Maladie rates.

Results: 23,152 pts with pSS and 15,462 with SS+AID were identified during the study period. Estimated prevalence of SS (2011-2018) per 100,000 persons ranged from 22-32 (pSS) and 17-22 (SS+AID) and was stable over time; proportion of female pts was 90-91\% (pSS) and 92-93\% (SS+AID); mean age ranged from 63-66 (pSS) and 58-63 (SS+AID) years. Estimated incidence of SS (2012-2018) per 100,000 persons ranged from 4.1-0.3 (pSS) and 2.1-0.1 (SS+AID); proportion of female pts was 76-87\% (pSS) and 74-90\% (SS+AID); mean age ranged from 53-64 (pSS) and 50-62 (SS+AID) years. RA was the most frequently associated autoimmune disorder (32\%) in pts with SS+AID. In the first year, artificial tears was the most frequently reimbursed drug (66\% [pSS], $55 \%$ [SS+AID]), followed by hydroxychloroquine (19\% [pSS], 35\% [SS+AID]). From $2014-2018, \sim 30 \%$ of men and $\sim 22 \%$ of women with pSS reported no drug reimbursement; $\sim 20 \%$ of men and $\sim 15 \%$ of women with SS+AID reported no drug reimbursement during the same time frame. From 2013-2018, 50\% of all pts had consultations with a dentist or an ophthalmologist. Mean healthcare costs increased over the study period: from €5836 to €9618 (pSS) and €8905 to $€ 13,271$ (SS+AID). Pt characteristics and healthcare consumption data are presented (Table 1).

Conclusion: Prevalence of pSS in this French claims data study was stable over time and consistent with previous reports. ${ }^{3}$ Based on these results, SS may be considered an orphan disease. Overall costs for pts with SS were higher than previously estimated. ${ }^{5}$ Results may help inform and optimise specialist care for pts with SS in France.

REFERENCES:

[1] Patel R, Shahane A. Clin Epidemiol 2014;6:247-255

[2] Mariette X, Criswell LA. N Engl J Med 2018;378:931-939.

[3] Maldini C, et al. Arthritis Care Res (Hoboken) 2014;66:454-463.

[4] Devauchelle Pensec V, et al. Arthritis Rheumatol 2019;71(suppl 10):abstract 1101.

[5] l'Assurance Maladie. Améliorer la qualité du système de santé et maîtriser les dépenses. July 2019

Acknowledgements: This study was sponsored by Bristol Myers Squibb. Professional medical writing assistance was provided by Lindsay Craik at Caudex and was funded by Bristol Myers Squibb.

Disclosure of Interests: Raphaèle Seror Consultant of: Amgen, Boehringer Ingelheim, Bristol Myers Squibb, Fresenius Kabi, GlaxoSmithKline, Janssen, Pfizer, Roche, Laurent Chiche: None declared, Guillaume Desjeux: None declared, Joe Zhuo Shareholder of: Bristol Myers Squibb, Employee of: Bristol Myers Squibb, Bruno Bregman Shareholder of: Bristol Myers Squibb, Employee of: Bristol Myers Squibb, Virginie Vannier-Moreau Shareholder of: Bristol Myers Squibb, Employee of: Bristol Myers Squibb, Valerie Devauchelle-Pensec Consultant of: AbbVie, Bristol Myers Squibb, Novartis, Grant/research support from: Bristol Myers Squibb, Lilly, Roche-Chugai.

DOI: 10.1136/annrheumdis-2021-eular.78

Table 1. Characteristics and healthcare consumption data for pts with SS in the SNDS (2011-2018)

pss

SS+AID

B. G. Soussi ${ }^{1}$, R. L. Cordtz ${ }^{1}$, S. Kristensen ${ }^{1,2}$, C. S. Bork ${ }^{3}$, J. Christensen ${ }^{2,4}$, E. B. Schmidt ${ }^{2,3}$, D. Prieto-Alhambra ${ }^{5}$, L. Dreyer ${ }^{1,2}$. 'Aalborg University Hospital, Department of Rheumatology, Aalborg, Denmark; ${ }^{2}$ Aalborg University, Department of Clinical Medicine, Aalborg, Denmark; ${ }^{3}$ Aalborg University Hospital, Department of Cardiology, Aalborg, Denmark; ${ }^{4}$ Aalborg University Hospital, Department of Nephrology, Aalborg, Denmark; ${ }^{5}$ University of Oxford, CSM-NDORMS, Oxford, United Kingdom

Background: Few previous studies have investigated the incidence rate (IR) and point prevalence (PP) of seropositive and seronegative rheumatoid arthritis (RA), and further, the estimates remain unknown in the Danish population.

Objectives: To investigate the IR and PP of seropositive and seronegative RA in the adult Danish population from 1998 to 2018 using four register-based case definitions of RA.

Methods: Nationwide register-based cohort study. Using the Danish administrative registers, patients with RA between 1998 and end of 2018 were identified. ICD-10 codes for RA were identified in the Danish National Patient Registry and information on DMARD prescriptions were obtained through the Danish National Prescription Registry using ATC codes. The used case definitions were ${ }^{1}$ : Criteria A, first time M05/M06 RA diagnosis and redemption of a DMARD in the following year; Criteria $B$, two RA diagnoses within 90 days of each other, originating from department of rheumatology or general internal medicine, where the latest registered M05/M06 diagnosis defined the serologic status; Criteria C, a M05/M06 diagnosis recorded at any time preceded or followed by redemption of a DMARD prescription within one year, where the M05/M06 diagnosis recorded determined the serologic status; Criteria $D$, as Criteria $A$, but with the additional requirements that cases had no registration for other selected inflammatory diseases.

In calculation of IRs the total Danish population from 1998 to 2018 was used as reference population for standardisation. The PP was calculated for years 2000 , 2009, 2011 and 2018.

Results: From 1998 to 2018 the overall IR was $18.0(95 \% \mathrm{Cl} 17.7$ to 18.3$)$ per 100,000 person years (PY) for seropositive RA and $16.7(95 \% \mathrm{Cl} 16.4$ to 16.9$)$ per 100,000 PY for seronegative RA using Criteria A. A higher IR for seropositive RA than for seronegative RA was found regardless of the case criteria used. Figure 1 shows the temporal IRs of seropositive and seronegative RA

Regardless of case criteria used, the PP increased from 2000 to 2018 for both seropositive and seronegative RA, and the estimates were higher for seropositive RA than for seronegative RA (Table 1).

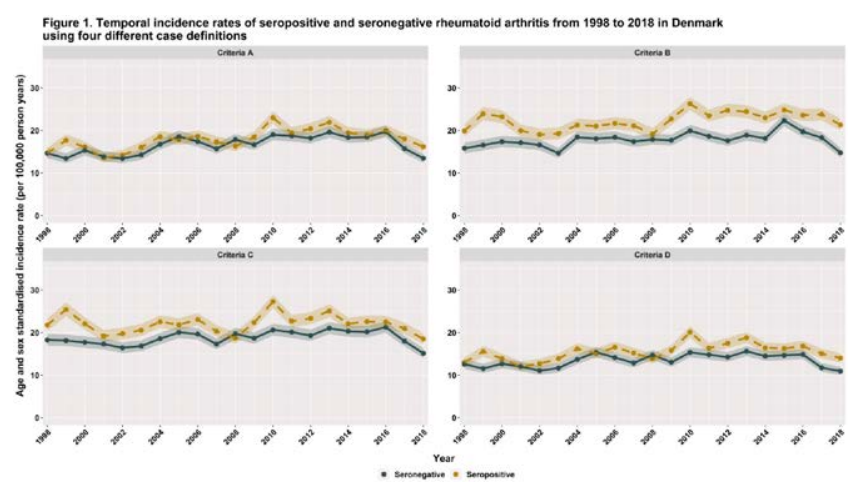

Age at disease onset, overall and by sex

\begin{tabular}{|c|c|c|c|c|c|c|}
\hline & $M(n=2515)$ & $F(n=20,637)$ & All $(n=23,152)$ & $M(n=1350)$ & $F(n=14,112)$ & All $(n=15,462)$ \\
\hline Mean (SD) & $62(15)$ & $60(15)$ & $60(15)$ & $59(14)$ & $56(15)$ & $56(15)$ \\
\hline \multicolumn{7}{|l|}{ Age, yrs, n (\%) } \\
\hline$<50$ & $480(19)$ & $4948(24)$ & $5428(23)$ & $328(24)$ & 4507 (32) & $4835(31)$ \\
\hline $50-74$ & $1471(59)$ & $11,567(56)$ & $13,038(56)$ & $852(63)$ & $7887(56)$ & $8739(57)$ \\
\hline $\begin{array}{l}\geq 75 \\
\text { Healthcare consumption }\end{array}$ & $564(22)$ & $4122(20)$ & $4686(20)$ & $170(13)$ & $1718(12)$ & $1888(12)$ \\
\hline Mean (IQR) no. prescriptions for treatment of interest & & $37(9-58)$ & & & $52(20-78)$ & \\
\hline$\geq 1$ Schirmer's test, $\mathrm{n}(\%)$ & & $2409(10)$ & & & $1303(8)$ & \\
\hline Reimbursement for antinuclear antibody test, $\mathrm{n}(\%)$ & & $13,312(58)$ & & & $9587(62)$ & \\
\hline Mean no. dental reimbursements/yr & & 2 & & & 1 & \\
\hline Mean no. ophthalmologist reimbursements/yr & & 1 & & & 1 & \\
\hline
\end{tabular}

$\mathrm{F}=$ female; $\mathrm{IQR}=$ interquartile range; $\mathrm{M}=$ male; $\mathrm{yr}=\mathrm{year}$ 